\title{
Repercussões do processo de reestruturação dos serviços de saúde mental para crianças e adolescentes na cidade de Campinas, São Paulo (2006-2011)
}

\author{
Analysis of the impact of reorganization \\ of mental health services for children \\ and adolescents in Campinas, \\ São Paulo (2006-2011)
}

\author{
Melissa Ribeiro TEIXEIRA ${ }^{1}$ \\ Maria Cristina Ventura COUTO ${ }^{1}$ \\ Pedro Gabriel Godinho DELGADO'
}

\begin{abstract}
Resumo
Este estudo descreve e analisa as mudanças realizadas na rede de Saúde Mental para Crianças e Adolescentes da cidade de Campinas, São Paulo, no período de 2006 a 2011, durante o qual foram realizadas diferentes ações para reorientação do cuidado. Com foco nos Centros de Atenção Psicossocial, o artigo discute questões relacionadas ao acesso e aos fluxos de atendimento, permitindo identificar alguns avanços e desafios resultantes da reestruturação da rede de Saúde Mental para Crianças e Adolescentes do município. Trata-se de estudo exploratório, realizado através de coleta de informações e dados em prontuários e documentos de dois serviços de Saúde Mental para Crianças e Adolescentes de Campinas e um documento da gestão municipal. Constatou-se que a reestruturação propiciou maior articulação com a atenção primária de saúde, avanço na organização de base territorial e diminuição relativa dos encaminhamentos originados do setor justiça/direitos. Novos estudos são necessários para avaliar o impacto das mudanças na ampliação do acesso ao tratamento em Saúde Mental para Crianças e Adolescentes.
\end{abstract}

Palavras-chave: Adolescente; Atenção primária à saúde; Criança; Saúde mental; Serviço de saúde mental.

\begin{abstract}
This paper aims to describe and analyze the changes made in child and adolescent mental health services in Campinas, São Paulo between 2006 and 2011. During this time period, different actions were taken to reorganize the health care services provided. Focusing on the Centers for Psychosocial Care, this exploratory research addresses issues

$\because \cos$

1 Universidade Federal do Rio de Janeiro, Instituto de Psiquiatria, Núcleo de Pesquisa em Políticas Públicas de Saúde Mental. Av. Venceslau Brás, 71, Fundos, Botafogo, 22290-140, Rio de Janeiro, RJ, Brasil. Correspondência para/Correspondence to: M.R. TEIXEIRA.

E-mail: <melissartos@gmail.com>.
\end{abstract}


related to the access and delivery of health care in order to identify the improvements and challenges resulting from the reorganization of the health services. Data and information were collected from medical records and documents in two child and adolescent mental health services. It was found that the reorganization process provided better liaison with the primary care, led to improvements in the community-based organization, and caused a relative decrease in the referrals from the justice system. Further studies are necessary to assess the impact of these changes made to improve the access to mental health care for children/adolescents.

Keywords: Adolescent; Primary health care; Child; Mental health; Mental health services.

Apesar da magnitude dos problemas de Saúde Mental em Crianças e Adolescentes (SMCA), apenas recentemente, nos contextos mundial e nacional, estão sendo desenvolvidas ações mais consistentes em prol da organização de redes de serviços com condições de responder às diferentes ordens de questões implicadas na SMCA (Belfer, 2008; Couto, Duarte, \& Delgado, 2008; World Health Organization [WHO], 2005). No Brasil, somente em 2002 o Sistema Único de Saúde (SUS), através da Portaria n 336/02 (Brasil, 2002a), propôs orientação técnica e destinou recursos financeiros para a implantação de Centros de Atenção Psicossocial Infantojuvenis (CAPSi), inaugurando a primeira ação concreta da saúde mental no SUS para crianças e adolescentes.

Esses serviços são destinados ao cuidado dos casos de maior gravidade e complexidade, e devem, além da função de tratamento, responsabilizar-se pela ordenação das diferentes demandas e da rede de SMCA de seu território (Brasil, 2002b; Delfini \& Reis, 2012). Assim, torna-se possível ampliar o acesso de crianças e adolescentes com necessidades em saúde mental e construir, juntamente com demais dispositivos de saúde e em colaboração intersetorial, a rede local de cuidados. Além dos CAPSi, a articulação da saúde mental com os setores historicamente envolvidos na assistência à infância e adolescência - como o da saúde geral, educação, assistência social, justiça/direitos -, vem sendo afirmada como a principal estratégia para a construção no país de uma rede ampliada de atenção em SMCA baseada no fundamento intersetorial (Couto et al., 2008).

A necessidade de construir redes ampliadas de cuidado em SMCA de base territorial e articuladas a partir dos CAPSi, foi deliberada na III Conferência Nacional de Saúde Mental (CNSM), realizada na IV CNSM de 2010 (Brasil, 2010) como forma de superar a desassistência a que estavam submetidas, até então, crianças e adolescentes com problemas de saúde mental.

Até o final do século $X X$, o cenário brasileiro em relação à SMCA foi marcado pela desassistência. Esta é traduzida principalmente pela tendência à institucionalização de crianças e adolescentes em abrigos e educandários de cunho estritamente corretivo-disciplinar (Couto, 2004; 2012; Rizzini, 2010) situados fora do sistema de saúde, e pelo não reconhecimento da possibilidade de sofrimento e adoecimento psíquico em crianças e jovens. A insígnia de deficiência encobriu grande parte do problema, contribuindo para adiar a formação de uma agenda pública voltada para a montagem de redes de cuidados sensíveis aos diferentes modos de sofrer e padecer que podem marcar a vida de muitas crianças e jovens.

O acesso, isto é, "o grau de facilidade com que as pessoas obtêm cuidados de saúde" (Travassos \& Martins, 2004, p.191), é um componente indispensável para assegurar a qualidade na atenção à saúde. A estruturação das redes de atenção deve buscar propiciar o acesso com qualidade. Estudo sobre a atenção à criança e redes de saúde, comparando configurações que têm como eixo estruturante a Estratégia de Saúde da Família (ESF) em relação a redes com baixa integração com a atenção básica, evidencia que um melhor acesso é obtido nas redes com articulação intrasetoral, especialmente com a ESF (Prado, Fujimori, \& Cianciarullo, 2007).

No município de Campinas (SP), há poucos anos, a situação da SMCA não diferia do panorama nacional no que diz respeito à existência de uma significativa lacuna entre as necessidades em saúde mental de crianças e adolescentes e a oferta de 
serviços para responder a elas (Amstalden, Hoffmann, \& Monteiro, 2010; Couto et al., 2008). No entanto, esses dados contrastavam com a densidade da rede de cuidados voltada para o atendimento da população adulta na mesma cidade (Figueiredo \& Campos, 2009).

Com cerca de 1.100.000 habitantes e área geográfica de 794,571 km² (Instituto Brasileiro de Geografia e Estatística, 2010) no ano de 2008, a cidade contava apenas com dois serviços públicos para atendimento em SMCA, que operavam de forma centralizada, organizavam o trabalho a partir de critérios pouco inclusivos - como perfil diagnóstico e recorte de faixa etária -, e eram desconectados das reais necessidades do território. Esse cenário acabou por gerar resultados pouco efetivos na ampliação do acesso e qualificação do cuidado em SMCA (Prefeitura Municipal de Campinas, 2008). O primeiro, um Centro de Referência em Saúde Mental para Adolescentes (Serviço 1), tinha como público alvo apenas adolescentes em uso abusivo de álcool e drogas e em vulnerabilidade social. O segundo, mesmo habilitado no SUS como CAPSi (Serviço 2), restringia o atendimento aos casos de autismo e psicose infantil, desconsiderando outras necessidades em seu território, sem cumprir, portanto, as orientações técnicas da Portaria n 336/02 (Brasil, 2002a). A despeito da importância do trabalho realizado nesses serviços, eles não foram capazes de alterar positivamente os dados de acesso e cobertura em SMCA no município, que permaneciam aquém das reais necessidades territoriais (Prefeitura Municipal de Campinas, 2009).

A necessidade de reestruturar a atenção em SMCA na cidade de Campinas (SP) fez com que, em 2009, fosse realizada uma Oficina de Trabalho, a qual contou com a participação de gestores e profissionais de saúde mental. O principal objetivo era construir um amplo e aprofundado diagnóstico da situação no município e propor estratégias para reorganizar os serviços existentes, ampliar a base das articulações intra e intersetoriais e, desse modo, iniciar a construção de uma rede efetiva de atenção em SMCA. A necessidade de reestruturação imediata dos dois serviços, de modo que passassem a funcionar integralmente como CAPSi (Prefeitura Municipal de Campinas, 2009) foi consenso entre os participantes da Oficina. Assim, foi estabelecido que esses serviços reformulassem seus processos de trabalho para efetivamente operarem como dispositivos territoriais de atenção psicossocial, em estreita conexão com as necessidades do território, abertos às parcerias institucionais e setoriais necessárias ao melhor cuidado da infância e adolescência e em integração com a rede de saúde e de saúde mental da cidade. Para garantir essa reformulação, foi proposto ao Serviço 1 "a redefinição do modelo de assistência e do território" e, para o Serviço 2 "a ampliação da equipe, visando acompanhar o processo de reestruturação da rede de saúde mental da criança e do adolescente" (Prefeitura Municipal de Campinas, 2009).

A partir de 2010, o Serviço 1 passou a funcionar como um CAPSi, cobrindo uma área geográfica específica, correspondendo a 50\% da área do município; já o Serviço 2 foi reestruturado para realmente cumprir o papel de um CAPSi, sem restrição da clientela a quadros psicopatológicos específicos, responsabilizando-se pela cobertura da área restante da cidade.

O presente estudo visa descrever e analisar as mudanças na rede de SMCA de Campinas, no período de 2006 a 2011, especialmente as relacionadas à ampliação do acesso e à reorientação do fluxo do atendimento. Foi analisado, também, o perfil sociodemográfico da clientela atendida, com base nos dados oriundos dos prontuários e livros de registro. Além disso, a ampliação do acesso foi relacionada com o quantitativo de usuários em cada serviço em três períodos delimitados, e o fluxo de atendimento com a origem/procedência dos usuários atendidos nos dois serviços. A relevância dessas variáveis para análise das mudanças na rede campineira de SMCA tem relação com a hipótese de que a maior vinculação territorial dos serviços aumenta a cobertura, altera a cartografia do fluxo de atendimentos e adensa a parceria da SMCA com dispositivos da rede básica de saúde e da rede de educação, qualificando as principais vias de acesso ao cuidado. 


\section{Método}

Este é um estudo exploratório-descritivo, de base quantitativa, a partir de análise documental, realizado através de coleta de informações e dados em prontuários e documentos dos dois serviços de SMCA existentes na cidade de Campinas (SP), no período de 2006-2011, e que visa, especialmente, informações relacionadas às características sociodemográficas da clientela, origem/procedência dos usuários e situação do tratamento.

\section{Participantes}

A investigação se deu através de levantamento de dados de prontuários de 120 crianças e adolescentes que foram atendidos, ou ainda permaneciam em atendimento, nos dois serviços de SMCA de Campinas (SP), entre os anos de 2006 e 2011.

\section{Instrumentos}

Para coleta das informações foi realizado um levantamento dos prontuários do período estudado. Foram encontrados 1499 prontuários, sendo 1091 correspondentes ao Serviço 1, e 408 ao Serviço 2. Esse universo serviu de base para a caracterização sociodemográfica da clientela e para o levantamento da origem/procedência dos encaminhamentos aos serviços. Foi também utilizado o documento da gestão municipal de saúde, que descrevia as pactuações realizadas na Oficina de 2009 sobre o modelo de atenção em saúde mental da criança e adolescente, que determinaram a reestruturação da rede de serviços.

\section{Procedimentos}

Foram analisados dados oriundos de dois tipos de documentos: prontuários e livros de registro de abertura de prontuários dos dois serviços, a partir dos quais foram obtidos dados quantitativos da clientela atendida. Além dessas fontes de informação analisou-se, também, o registro da Oficina sobre Modelo de Atenção com a finalidade de obter a descrição das medidas de gestão pactuadas e que vieram a constituir o processo de reestruturação analisado. Estudos com base em documentos apresentam limitações e dificuldades, especialmente quando constituem, como no presente estudo, a integralidade do corpus de pesquisa (Bauer \& Gaskell, 2008). As fontes documentais utilizadas são, entretanto, valiosas, especialmente no contexto de baixa consolidação do processo de registro de informações de gestão da saúde, que ainda caracteriza o SUS e, especialmente, os serviços de SMCA no Brasil.

O período de tempo estipulado para o estudo atende ao objetivo de permitir a análise da situação antes e depois das reestruturações propostas em 2009, tendo sido subdividido em três: Período Anterior, de 2006 a 2007, antes da reestruturação; Período Intermediário, de 2008 a 2009, durante o processo de reestruturação; e Período Posterior, de 2010 a 2011, após a reestruturação. Além disso, foi afastada a ocorrência de repetições de registros, através da anotação das duplicidades, caso em que o registro duplicado foi excluído.

Considerando a proporcionalidade da clientela em cada serviço nos três períodos estabelecidos, foram escolhidos aleatoriamente 120 prontuários (90 prontuários do Serviço 1, e 30 do Serviço 2). Estes serviram de base para a análise da situação do tratamento, compreendida através das categorias de usuários com projeto terapêutico em andamento, usuários na situação de abandono do tratamento, usuários com alta clínica (exceto abandono ou ocorrência de óbito) ou encaminhados a outros serviços da cidade. Os critérios definidos para considerar abandono de tratamento foram: registro em prontuário do evento abandono ou ausência de registro em prontuário transcorridos 3 meses do último atendimento. A pesquisa foi aprovada pelo Comitê de Ética em Pesquisa do Instituto de Psiquiatria da Universidade Federal do Rio de Janeiro (Parecer $n^{\circ}$ 116.227, CAAE 06458412.2.0000.5263) e pela Secretaria Municipal de Saúde da Prefeitura de Campinas.

\section{Resultados}

O quantitativo de 1499 crianças e adolescentes foi atendido nos dois serviços de SMCA da 
cidade de Campinas (SP) entre os anos de 2006 e 2011. Considerando o número de pessoas atendidas por período, foram registrados 459 usuários no Período Anterior (Serviço $1=382$; Serviço $2=77$ ), 579 no Período Intermediário (Serviço $1=441$; Serviço 2 = 138) e 461 no Período Posterior (Serviço 1 = 268; Serviço 2 = 193). Os números referem-se aos usuários novos em cada serviço por período estudado, ou seja, somente aqueles registrados através da abertura de prontuário.

Do total de crianças e adolescentes, 1047 $(69,9 \%)$ eram do sexo masculino e $452(30,1 \%)$ do feminino. Quando analisados por Período - Anterior, Intermediário e Posterior à reestruturação na SMCA -, verificou-se que a porcentagem de usuários do sexo masculino aumentou após a reestruturação, como explicitado na Figura 1. Enquanto no Período Anterior $63,6 \%$ eram pacientes do sexo masculino e $36,4 \%$ do feminino, no Posterior esses números se modificaram, correspondendo a $75,3 \%$ e $24,7 \%$, respectivamente.

Na relação entre sexo e faixa etária, conforme demonstrado na Tabela 1, houve um predomínio de usuários entre 15 e 19 anos (42,8\%). Considerando os dados por período, registrou-se uma mudança importante na faixa etária de usuários do sexo feminino: no Período Anterior a maior parte estava na faixa de 10 a 14 anos (45,5\%); nos Períodos Intermediário e Posterior, a maioria situava-se entre 15 e 19 anos (Tabela 1).
No que diz respeito à origem/procedência dos pacientes, de acordo com o livro de registro de prontuários, foram identificadas instituições de diferentes setores públicos que mais frequentemente encaminhavam usuários para os dois serviços de SMCA. A ausência desse tipo de informação nos registros do Serviço 2 determinou que apenas os dados do Serviço 1 pudessem ser apresentados e analisados (Tabela 2).

Conforme apresentado na Tabela 2, nos Períodos Anterior e Intermediário o principal encaminhador foi o Conselho Tutelar, recobrindo 29,6\% da demanda no Período Anterior à reestruturação e $27,9 \%$ durante o processo de mudança. A Atenção Básica representou apenas 16,5\% dos enca-

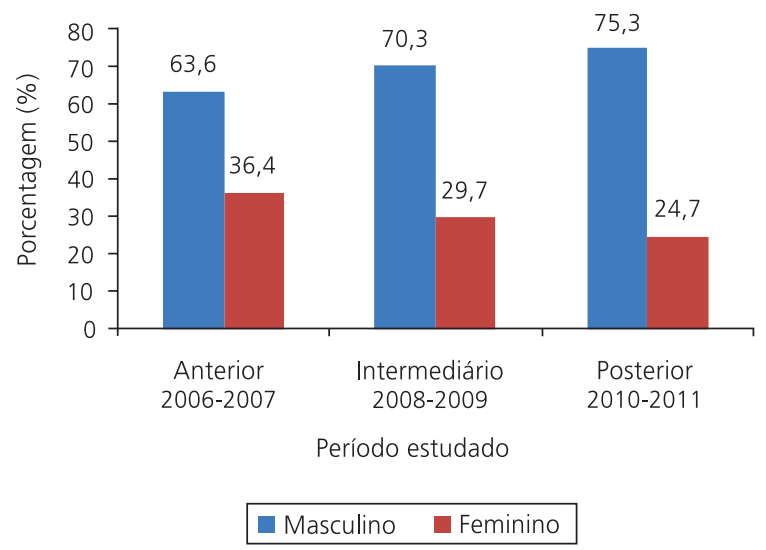

Figura 1. Distribuição de usuários, por sexo e período, nos serviços de atenção psicossocial infantojuvenil de Campinas (SP), 2006 a 2011 $(n=1499)$.

Tabela 1

Caracterização dos usuários dos serviços de atenção psicossocial infantojuvenil de Campinas (SP), segundo sexo e idade, por período, 2006 a 2011 $(n=1499)$

\begin{tabular}{|c|c|c|c|c|c|c|c|c|c|c|c|c|c|c|}
\hline \multirow{3}{*}{ Faixa Etáríodo e sexo } & \multicolumn{4}{|c|}{ Anterior 2006 - 2007} & \multicolumn{4}{|c|}{ Intermediário 2008 - 2009} & \multicolumn{4}{|c|}{ Posterior 2010 - 2011} & \multirow{2}{*}{\multicolumn{2}{|c|}{ Total }} \\
\hline & \multicolumn{2}{|c|}{ Masculino } & \multicolumn{2}{|c|}{ Feminino } & \multicolumn{2}{|c|}{ Masculino } & \multicolumn{2}{|c|}{ Feminino } & \multicolumn{2}{|c|}{ Masculino } & \multicolumn{2}{|c|}{ Feminino } & & \\
\hline & $n$ & $\%$ & $n$ & $\%$ & $n$ & $\%$ & $\mathrm{n}$ & $\%$ & $n$ & $\%$ & $n$ & $\%$ & $\mathrm{n}$ & $\%$ \\
\hline 0 a 4 & 9 & 3,1 & 5 & 3,0 & 25 & 6,1 & 2 & 1,2 & 26 & 2,5 & 5 & 4,4 & 72 & 4,8 \\
\hline 5 a 9 & 21 & 7,2 & 7 & 4,2 & 35 & 8,6 & 14 & 8,2 & 54 & 5,2 & 14 & 12,3 & 145 & 9,7 \\
\hline 10 a 14 & 115 & 39,4 & 76 & 45,5 & 146 & 35,8 & 68 & 39,8 & 122 & 35,2 & 40 & 35,1 & 567 & 37,8 \\
\hline 15 a 19 & 134 & 45,9 & 65 & 38,9 & 182 & 44,6 & 78 & 45,6 & 135 & 38,9 & 48 & 42,1 & 642 & 42,8 \\
\hline 20 ou mais & 0 & 0,0 & 0 & 0,0 & 0 & 0,0 & 0 & 0,0 & 0 & 0,0 & 1 & 0,9 & 1 & 0,1 \\
\hline Não informado & 13 & 4,5 & 14 & 8,4 & 20 & 4,9 & 9 & 5,3 & 10 & 2,9 & 6 & 5,3 & 72 & 4,8 \\
\hline Total & 292 & 100,0 & 167 & 100,0 & 408 & 100,0 & 171 & 100,0 & 347 & 84,6 & 114 & 100,0 & 1499 & 100,0 \\
\hline
\end{tabular}

Nota: *Foi considerada a divisão de faixa etária do Instituto Brasileiro de Geografia e Estatística. 
Tabela 2

Origem/procedência dos usuários do serviço de atenção psicossocial infantojuvenil de Campinas (SP) (Serviços 1), por período, 2006 a 2011 $(n=1091)^{*}$

\begin{tabular}{|c|c|c|c|c|c|c|}
\hline \multirow[b]{2}{*}{ Origem } & \multicolumn{2}{|c|}{ Anterior 2006 - 2007} & \multicolumn{2}{|c|}{ Intermediário 2008 - 2009} & \multicolumn{2}{|c|}{ Posterior 2010 - 2011} \\
\hline & $\mathrm{n}$ & $\%$ & $\mathrm{n}$ & $\%$ & $\mathrm{n}$ & $\%$ \\
\hline Atenção Básica & 63 & 16,5 & 70 & 15,9 & 126 & 47,0 \\
\hline Hospital geral & 19 & 5,0 & 21 & 4,8 & 19 & 7,1 \\
\hline Outros serviços de saúde & 15 & 3,9 & 18 & 4,1 & 34 & 12,7 \\
\hline Conselho Tutelar & 113 & 29,6 & 123 & 27,9 & 14 & 5,2 \\
\hline Vara da Infância & 3 & 0,8 & 18 & 4,1 & 4 & 1,5 \\
\hline Medida sócioeducativa & 25 & 6,5 & 59 & 13,4 & 21 & 7,8 \\
\hline Assistência Social & 59 & 15,4 & 35 & 7,9 & 16 & 6,0 \\
\hline Escola & 7 & 1,8 & 4 & 0,9 & 2 & 0,7 \\
\hline Espontâneo & 43 & 11,3 & 57 & 12,9 & 20 & 7,5 \\
\hline Outros & 26 & 6,8 & 26 & 5,9 & 6 & 2,2 \\
\hline Não informado & 9 & 2,4 & 10 & 2,3 & 6 & 2,2 \\
\hline Total & 382 & 100,0 & 441 & 100,0 & 268 & 100,0 \\
\hline
\end{tabular}

Nota: *Do total de pacientes recebidos pelos dois serviços $(n=1499)$, somente foi possível identificar a origem de encaminhamentos dos pacientes do Serviço 1, pois no Serviço 2 não consta esta informação no livro de registro de prontuários.

minhamentos no Período Anterior, seguida pela Assistência Social (15,4\%), e 15,9\% no período Intermediário, seguida pelas instituições de cumprimento de Medidas Socioeducativas (13,4\%).

No Período Posterior, entretanto, $47,0 \%$ dos encaminhamentos passaram a ser referenciados pela Atenção Básica, seguidos por outros Serviços de Saúde, com 12,7\%. Nesse período, os encaminhamentos das instituições do setor Justiça/Direitos diminuíram significativamente: apenas 7,8\% foram originados das instituições de cumprimento de Medidas Socioeducativas, 5,2\% do Conselho Tutelar e 1,5\% da Vara da Infância e Juventude.

Os resultados relacionados à situação do tratamento - dados coletados em 120 prontuários dos Serviços 1 e 2, segundo proporcionalidade por período -, indicaram ter havido uma significativa alteração no percentual de usuários mantidos em atendimento nos serviços depois da reestruturação proposta pelos gestores e trabalhadores da saúde mental no ano de 2009 (Figura 2).

Antes da reestruturação, a maioria dos registros indicava a situação de abandono do tratamento (67,5\% no Período Anterior) ou de encaminhamento para outros serviços da cidade $(20,0 \%$ dos casos). No Período Posterior, os dados indicam uma inversão, ou seja, um aumento expressivo de

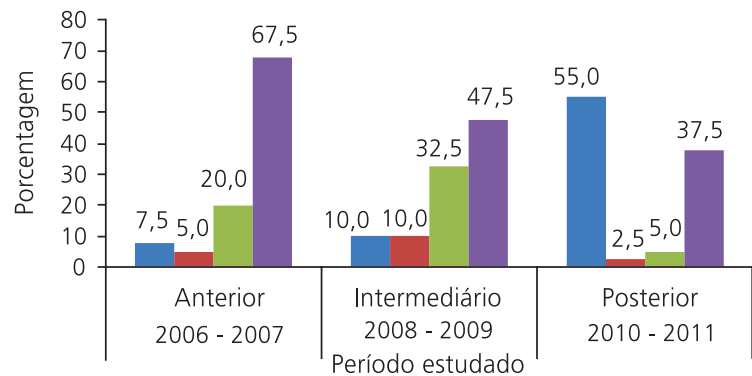

a em tratamento $\square$ alta encaminhamento $\mathbf{a b a n d o n o}$

Figura 2. Situação do tratamento, por período, nos serviços de atenção psicossocial infantojuvenil de Campinas (SP), 2006 a 2011 $(n=120)$.

usuários com projeto terapêutico em andamento $(55,0 \%)$ e uma diminuição significativa do abandono $(37,5 \%)$.

\section{Discussão}

A análise da reestruturação dos serviços de SMCA de Campinas permitiu identificar alguns avanços e desafios na organização da rede de SMCA do município. O primeiro ponto a destacar se refere à maior presença da Atenção Básica na demanda ao CAPSi após as mudanças propostas em 2009. Estudos sobre a construção de sistemas de cuidado em SMCA vêm enfatizando a importância da Aten- 
ção Básica na ampliação do acesso, tanto em países desenvolvidos como em desenvolvimento, pela maior proximidade entre esses serviços e a população, bem como pelo caráter menos estigmatizante que representam na busca por cuidado, quando comparados aos serviços específicos de SMCA (Paula, Lauridsen-Ribeiro, Wissow, Bordin, \& Evans-Lacko, 2012; WHO, 2005).

Após o período de reestruturação (2010-2011), nomeado aqui como Período Posterior, a Atenção Básica passou a representar o principal encaminhador, tendo em vista que antes (2006-2007) o principal era o Conselho Tutelar. Essa mudança sugere que os serviços, ao operarem como dispositivos de base comunitária articulados aos territórios, vinculam-se mais fortemente com a Atenção Básica e podem redesenhar o itinerário da busca por atendimento em SMCA, diminuindo a possível judicialização do cuidado - fato ocorre quando há intervenção do judiciário ou de órgãos de defesa de direitos para a efetivação da assistência a uma dada população.

A vinculação com a Atenção Básica pode, ainda, qualificar e dotar de maior efetividade os encaminhamentos, incidindo sobre a adesão ao tratamento. Paim, Travassos, Almeida, Bahia e Macinko (2011) ressaltam que os usuários da ESF percebem efeitos positivos de acolhimento e adesão quando os encaminhamentos para outros serviços da rede são feitos por uma equipe da ESF. No caso da SMCA, a articulação com a Atenção Básica pode, também, favorecer o compartilhamento e a corresponsabilização dos casos, ações que "garantem maior resolutividade no manejo de situações que envolvem sofrimento psíquico" (Delfini \& Reis, 2012, p.358) em crianças e adolescentes.

As ações conjuntas com a Atenção Básica devem se constituir como estratégias fundamentais para a consolidação da rede de SMCA, principalmente no que tange à adesão e continuidade do tratamento. Da mesma forma, o CAPSi, como ordenador da demanda desses pacientes em seu território, deve se fazer presente e diretamente implicado na articulação e proposição das ações de cuidado quer junto à rede básica, quer junto às demais instituições que cuidam da infância e adolescência -, através da posição decidida de estabelecer como comum um problema que atravessa e implica diferentes setores (Couto \& Delgado, 2010, p.278), mas que, frequentemente, é tomado como restrito à intervenção do campo especializado.

Chamou a atenção nos dados coletados a baixa presença da rede de Educação na origem/procedência dos encaminhamentos, nos três períodos estudados. Política universal para crianças e adolescentes, e espaço privilegiado para as primeiras experiências de socialização e de ampliação de laços, seria esperado da escola maior frequência nas demandas para esses serviços. Os limites da investigação desenvolvida no presente estudo não permitem uma análise mais aprofundada sobre esse achado, mas sugerem a necessidade de estudos específicos que possam esclarecer seus possíveis determinantes. A articulação dos dispositivos da SMCA com a Saúde Geral, Educação, Assistência Social, Justiça/Direitos, bem como com demais setores envolvidos no cuidado de crianças e adolescentes, precisa ser priorizada como estratégia fundamental para a ampliação do acesso e consolidação da Rede Ampliada de Atenção à SMCA (Couto, 2012).

Embora não tenha ocorrido um aumento no número global de casos atendidos nos dois serviços nos períodos estudados, um aspecto importante registrado no processo de reestruturação da rede de SMCA em Campinas foi o aumento no percentual de casos em acompanhamento regular nos serviços e a diminuição do abandono ao tratamento, ambos registrados depois de 2009. É preciso ressaltar, no entanto, que o percentual de abandono depois das mudanças ainda foi expressivo, sugerindo a necessidade de revisão nas ações de cuidado contínuo realizadas pelas equipes de SMCA na cidade de Campinas.

É importante destacar que, também como efeito da reestruturação da rede, houve um aumento no número de casos acompanhados no Serviço 2, porém uma redução de usuários no Serviço 1. Esses dados podem sinalizar uma distribuição da demanda de SMCA entre os serviços, e a reorganização dos mesmos para operar como CAPSi. 
O perfil da clientela assistida nos dois CAPSi de Campinas coincide com os achados da literatura (Hoffmann, Santos, \& Mota, 2008; Reis, Delfini, Dombi-Barbosa, \& Oliveira, 2010; Santos, Carvalho, Pinho, \& Nunes, 2005), apontando para o predomínio da população masculina. A dominância de adolescentes (faixa etária dos 15 aos 19 anos) indica, ainda, a importância de maior articulação com serviços básicos de saúde e com as escolas, para ampliar condições de acesso ainda na infância de casos com agravos em saúde mental.

A reorientação dos serviços de SMCA no município mostrou ser uma estratégia importante, mesmo que inicial, de enfrentamento à desassistência e de reorganização da rede. A cidade ainda requer investimentos na oferta de novos serviços em SMCA e qualificação das articulações intra e intersetoriais, principalmente com o setor educacional. A reestruturação dos serviços não encerra em si o conjunto das ações necessárias ao melhor cuidado em SMCA, pois "o componente dinâmico e processual da rede - a direcionalidade do trabalho - é tão decisivo quanto o componente estrutural - a existência de serviços" (Couto, 2012, p.96).

A mudança no cenário da saúde pública e a reorientação dos serviços requerem uma visão crítica e abrangente das práticas em saúde mental, baseada na atuação interdisciplinar, trazendo novos desafios na identificação das necessidades em SMCA. O trabalho em equipe multidisciplinar e em colaboração intersetorial constitui um eixo fundamental de desenvolvimento da atenção psicossocial no território. Isso se constitui como um desafio para a atuação dos profissionais da saúde mental, inclusive o psicólogo, no que se refere ao trabalho na Atenção Básica à Saúde, como estratégia de promoção de cuidado em SMCA (Dimenstein, 1998; Paula, Vicente, \& Lowenthal, 2011).

Os resultados encontrados neste estudo apontam alguns efeitos da reestruturação da rede de SMCA de Campinas referentes à reorganização dos serviços e da relação destes com outros equipamentos, principalmente a Atenção Básica. O fortalecimento da rede intrasetorial promoveu fluxos assistenciais menos segmentados, fundamentais para a mudança e qualificação da linha de cuidado de saúde mental direcionada a crianças e adolescentes.

Por se tratar de um estudo exploratório, restrito ao levantamento documental, torna-se necessária a realização de novas pesquisas que desenvolvam uma análise aprofundada da rede de SMCA de Campinas, no intuito de avaliar a ampliação do acesso e a efetividade dos serviços a partir da nova configuração da rede. Um importante desafio a ser enfrentado diz respeito à necessidade de maior regularidade e qualidade no registro, organização dos dados e informações geradas pelos serviços de SMCA e pela rede intersetorial de cuidado. A produção de conhecimento sobre as inovações que vêm sendo operadas na rede de atenção psicossocial para crianças e adolescentes requer a organização e disseminação das informações pelos serviços, sejam elas de ordem epidemiológica, política ou clínica. Sem uma base consistente de registros, a possibilidade de análise e avaliação dos efeitos produzidos pelas diretrizes atuais ficará comprometida.

\section{Referências}

Amstalden, A. L. F., Hoffmann, M. C. C. L., \& Monteiro, T. P. M. (2010). A política de saúde mental infantojuvenil: seus percursos e desafios. In E. LauridsenRibeiro \& O. Y. Tanaka (Eds.), Atenção em saúde mental para crianças e adolescentes no SUS (pp.33-45). São Paulo: Hucitec.

Bauer, M. W., \& Gaskell, G. (Orgs.). (2008). Pesquisa qualitativa com texto, imagem e som: um manual prático ( $7^{\mathrm{a}}$ ed.). Petrópolis: Vozes.

Belfer, M. L. (2008). Child and adolescent mental disorders: The magnitude of the problem across the globe. Journal of Child Psychology and Psychiatry, 49(3), 226-236.

Brasil. Ministério da Saúde. (2002a). Portaria n 336, de 19 de fevereiro de 2002. Dispõe sobre as normas e diretrizes para a organização dos CAPS. Diário Oficial da União, p.22-23.

Brasil. Ministério da Saúde. (2002b). Relatório Final da III Conferência Nacional de Saúde Mental. Brasília: Ministério da Saúde.

Brasil. Ministério da Saúde. (2010). Relatório Final da IV Conferência Nacional de Saúde Mental Intersetorial. Brasília: Ministério da Saúde.

Couto, M. C. V. (2004). Por uma política pública de saúde mental para crianças e adolescentes. In T. Ferreira (Ed.), 
A criança e a saúde mental: enlaces entre a clínica e a política (pp.61-74). Belo Horizonte: Autêntica.

Couto, M. C. V. (2012). Política de saúde mental para crianças e adolescentes: especificidades e desafios da experiência brasileira (Tese de doutorado não-publicada). Universidade Federal do Rio de Janeiro.

Couto, M. C. V., \& Delgado, P. G. G. (2010). Intersetorialidade: exigência da clínica com crianças na atenção psicossocial. In E. Lauridsen-Ribeiro \& O. Y. Tanaka (Eds.), Atenção em saúde mental para crianças e adolescentes no SUS (pp.271-279). São Paulo: Hucitec.

Couto, M. C. V., Duarte, C. S., \& Delgado, P. G. G. (2008). A saúde mental infantil na saúde pública brasileira: situação atual e desafios. Revista Brasileira de Psiquiatria, 30(4), 390-398.

Delfini, P. S. S., \& Reis, A. O. A. (2012). Articulação entre serviços públicos de saúde nos cuidados voltados à saúde mental infantojuvenil. Cadernos de Saúde Pública, 28(2), 357-366.

Dimenstein, M. (1998). O psicólogo nas unidades básicas de saúde: desafio para a formação e atuação profissionais. Estudos de Psicologia (Natal), 3(1), 53-81.

Figueiredo, M. D., \& Campos, R. O. (2009). Saúde Mental na atenção básica à saúde de Campinas, SP: uma rede ou um emaranhado? Ciência \& Saúde Coletiva, 14(1), 129-138.

Hoffmann, M. C. C. L., Santos, D. N., \& Mota, E. L. A. (2008). Caracterização dos usuários e dos serviços prestados por Centros de Atenção Psicossocial infantojuvenil. Cadernos de Saúde Pública, 24(3), 633-42.

Instituto Brasileiro de Geografia e Estatística. (2010). Censo demográfico 2010: população por município. Brasília: Autor. Recuperado em março 2, 2011, de http:// www.ibge.gov.br/home/estatistica/populacao/censo 2010/tabelas_pdf/total_populacao_sao_paulo.pdf

Paim, J., Travassos, C., Almeida, C., Bahia, L., \& Macinko, J. (2011). O sistema de saúde brasileiro: história, avanços e desafios. Revista Lancet, 377(9779), 11-31.

Paula, C. S., Lauridsen-Ribeiro, E., Wissow, L., Bordin, I. A., \& Evans-Lacko, S. (2012). How to improve the mental health care of children and adolescent in Brazil:
Actions needed in public sector. Revista Brasileira de Psiquiatria, 34(3), 334-51.

Paula, C. S., Vicente, L. Z., \& Lowenthal, T. R. (2011). Atuação de psicólogos e estruturação de serviços públicos na assistência à saúde mental de crianças e adolescentes. Psicologia: Teoria e Prática, 13(3), 81-95.

Prado, S. R. L. A., Fujimori, E., \& Cianciarullo, T. I. (2007). A prática da integralidade em modelos assistenciais distintos: estudo de caso a partir da saúde da criança. Texto \& Contexto Enfermagem, 16(3), 399-407.

Prefeitura Municipal de Campinas. (2008). Secretaria Municipal de Saúde. Relatório anual de Gestão da SMS de 2008. Recuperado em março 2, 2011, de http:// 2009.campinas.sp.gov.br/saude/

Prefeitura Municipal de Campinas. (2009). Secretaria Municipal de Saúde. Relatório anual de Gestão da SMS de 2009. Recuperado em março 2, 2011, de http://2009.campinas.sp.gov.br/saude/

Reis, A. O., Delfini, P. S. S., Dombi-Barbosa, C., \& Oliveira, M. F. A. P. B. (2010). Crianças e adolescentes em sofrimento psíquico atendidos nos Centros de Atenção Psicossocial infantojuvenis. In E. Lauridsen-Ribeiro \& O. Y. Tanaka (Eds.), Atenção em Saúde Mental para crianças e adolescentes no SUS (pp.186-210). São Paulo: Hucitec.

Rizzini, I. (2010). Do confinamento ao acolhimento: institucionalização de crianças e adolescentes com deficiência: desafios e caminhos. Rio de Janeiro: Pontifícia Universidade Católica do Rio de Janeiro.

Santos, D. N., Carvalho, M. M., Pinho, M. A., \& Nunes, A. P. R. (2005). Atendimento em psiquiatria da infância e adolescência em serviços públicos de Salvador. Revista Baiana de Saúde Pública, 29(1), 35-42.

Travassos, C., \& Martins, M. (2004). Uma revisão sobre os conceitos de acesso e utilização de serviços de saúde. Cadernos de Saúde Pública, 20(Supl.2), 190-198.

World Health Organization. (2005). Atlas child and adolescent mental health resources: Global concerns implications for the future. Genebra: Author.

Recebido: março 3, 2013

Versão final: abril 16, 2014

Aprovado: maio 19, 2014 
